\title{
SECOND METHOD OF LYAPUNOV FOR STABILITY OF LINEAR IMPULSIVE DIFFERENTIAL- DIFFERENCE EQUATIONS WITH VARIABLE IMPULSIVE PERTURBATIONS
}

\author{
D.D. BAINOV \\ Higher Medical Institute, P.O. Box 45 \\ Sofia-1504, Bulgaria \\ I.M. STAMOVA \\ Technical University \\ Sliven, Bulgaria \\ A.S. VATSALA \\ University of Southwestern Louisiana \\ Department of Mathematics, P.O. Box 41010 \\ Lafayette, LA 70504, USA
}

(Received May, 1996; Revised June, 1997)

The present work is devoted to the study of stability of the zero solution to linear impulsive differential-difference equations with variable impulsive perturbations. With the aid of piecewise continuous auxiliary functions, which are generalizations of the classical Lyapunov's functions, sufficient conditions are found for the uniform stability and uniform asymptotical stability of the zero solution to equations under consideration.

Key words: Lyapunov Stability, Variable Impulses.

AMS subject classifications: $34 \mathrm{~A} 37,34 \mathrm{D} 20,34 \mathrm{~A} 30$.

\section{Introduction}

The impulsive differential-difference equations are adequate mathematical models of various real processes and phenomena that are characterized by rapid change of their state and dependence on the pre-history at each moment. In spite of great possibilities of applications, the theory of these equations is developing rather slowly due to difficulties of technical and theoretical character.

If the impulses are realized at fixed moments of time, the results can be easily derived by virtue of the corresponding result in the continuous case. Studies of impulsive differential-difference equations with variable impulsive perturbations carry lots of difficulties due to the presence of phenomena such as "beating" of the solutions, bi- 
furcation, loss of property of autonomy, etc. The importance of these equations in mathematical modeling necessitates to prove criteria for stability of their solutions.

The investigations of the present work are carried out with the aid of piecewise continuous Lyapunov's functions [3] and a technique that uses minimal subsets of suitable spaces of piecewise continuous functions. The elements of these subsets help us estimate the derivatives of piecewise continuous auxiliary functions [1, 2].

\section{The Statement of the Problem and Preliminary Notes}

Let $\mathbb{R}_{+}=[0, \infty) ; \mathbb{R}^{n}$ be the $n$-dimensional Euclidean space with elements $x=$ $\operatorname{col}\left(x_{1}, \ldots, x_{n}\right)$, the norm $|x|=\left(\sum_{k=1}^{n} s_{k}^{2}\right)^{1 / 2}$ and the scalar product $\langle x, y\rangle=$ $x_{1} y_{1}+\ldots+x_{n} y_{n}$. Let $h>0$ and $t_{0} \in \mathbb{R}$.

We consider the linear system of impulsive differential-difference equations

$$
\begin{gathered}
\dot{x}(t)=A(t) x(t)+B(t) x(t-h), \quad t \neq \tau_{k}(x(t)), t>t_{0}, \\
\left.\Delta x(t)\right|_{t=\tau_{k}(x(t))}=C_{k} x(t), t>t_{0}, \quad k=1,2, \ldots,
\end{gathered}
$$

where $x \in \mathbb{R}^{n}, A(t)$ and $B(t)$ are $n \times n$-matrix-valued functions; $C_{k}, k=1,2, \ldots$, are $n \times n$-matrices; $\tau_{k}: \mathbb{R}^{n} \rightarrow\left(t_{0}, \infty\right) ; \Delta x(t)=x(t+0)-x(t-0)$.

Let $\tau_{0}(x) \equiv t_{0}$ for $x \in \mathbb{R}^{n}$.

We introduce the following conditions:

H1. $\tau_{k} \in C\left[\mathbb{R}^{n},\left(t_{0}, \infty\right)\right], k=1,2, \ldots$.

H2. $t_{0}<\tau_{1}(x)<\tau_{2}(x)<\ldots, x \in \mathbb{R}^{n}$.

H3. $\tau_{k} \rightarrow \infty$ as $k \rightarrow \infty$ uniformly in $x \in \mathbb{R}^{n}$.

Assuming that conditions $\mathrm{H} 1, \mathrm{H} 2$, and $\mathrm{H} 3$ are satisfied, we introduce the following notations:

$$
\begin{gathered}
G_{k}=\left\{(t, x) \in\left[t_{0}, \infty\right) \times \mathbb{R}^{n}: \tau_{k-1}(x)<t<\tau_{k}(x)\right\}, \quad k=1,2, \ldots \\
\sigma_{k}=\left\{(t, x) \in\left[t_{0}, \infty\right) \times \mathbb{R}^{n}: t=\tau_{k}(x)\right\}
\end{gathered}
$$

i.e., $\sigma_{k}, k=1,2, \ldots$ are hypersurfaces with the equations $t=\tau_{k}(x(t))$.

Let $\varphi_{0} \in C\left[\left[t_{0}-h, t_{0}\right], \mathbb{R}^{n}\right]$.

We denote by $x(t)=x\left(t ; t_{0}, \varphi_{0}\right)$ the solution of system (1), (2) that satisfies the initial condition

$$
x(t)=\varphi_{0}(t), \quad t \in\left[t_{0}-h, t_{0}\right]
$$

The symbol $J^{+}\left(t_{0}, \varphi_{0}\right)$ stands for the maximal interval of the type $\left[t_{0}, \beta\right)$, at which the solution $x\left(t ; t_{0}, \varphi_{0}\right)$ is defined; $c_{0}=C\left[\left[t_{0}-h, t_{0}\right], \mathbb{R}^{n}\right]$, and $\|\varphi\|=$ $\max _{s \in\left[t_{0}-h, t_{0}\right]}|\varphi(s)|$ is the norm of the function $\varphi \in C_{0}$.

We will specify the solution $x(t)=x\left(t ; t_{0}, \varphi_{0}\right)$ of the initial problem (1), (2), (3) as follows:

1. For $t \in\left[t_{0}-h, t_{0}\right]$, the solution $x(t)$ coincides with the initial function $\varphi_{0}(t) \in$ $C_{0}$.

2. The function $x(t)$ is piecewise continuous on $J^{+}\left(t_{0}, \varphi_{0}\right), t=\tau_{k}(x(t)), t \neq \beta$, 
$k=1,2, \ldots$ and

3. For $t \in J^{+}\left(t_{0}, \varphi_{0}\right), t \neq \tau_{k}(x(t)), k=1,2, \ldots$, the function $x(t)$ is differentiable

$$
\dot{x}(t)=A(t) x(t)+B(t) x(t-h) .
$$

We make the following assumptions:

H4. The matrix-valued $n \times n$-functions $A(t)$ and $B(t)$ are continuous for $t \in$ $\left(t_{0}, \infty\right)$.

H5. $B(t)$ is a diagonal and $A(t)$ is antisymmetric matrix function.

H6. $C_{k}=\operatorname{diag}\left(c_{1 k}, \ldots, c_{n k}\right),-1<c_{i k} \leq 0, i=1, \ldots, n$.

H7. The integral curves of system (1), (2) meet successively each of the hypersurfaces $\sigma_{1}, \sigma_{2}, \ldots$ exactly once.

Condition $\mathrm{H} 7$ stipulate the absence of the "beating" phenomenon of the solutions to the system (1), (2), i.e., when a given integral curve meets more than once (or even infinitely many times) one and the same hypersurface. The "beating" phenomena is not present in the case when $\tau_{k}(x) \equiv t_{k}, k=1,2, \ldots, x \in \mathbb{R}^{n}$, i.e., when the impulses are realized at fixed moments.

Definition 1: The zero solution of system (1), (2) is said to be

a) uniformly stable, if

$$
\begin{gathered}
(\forall \epsilon>0)(\exists \delta=\delta(\epsilon)>0)\left(\forall t_{0} \in \mathbb{R}\right) \\
\left(\forall \varphi_{0} \in C_{0}:\left\|\varphi_{0}\right\|<\delta\right)\left(\forall t \in J^{+}\left(t_{0}, \varphi_{0}\right)\right): \\
\left|x\left(t ; t_{0}, \varphi_{0}\right)\right|<\epsilon ;
\end{gathered}
$$

b) uniformly attractive, if

$$
\begin{gathered}
(\exists \lambda>0)(\forall \epsilon>0) \quad(\exists \sigma=\sigma(\epsilon)>0) \quad\left(\forall t_{0} \in \mathbb{R}\right) \\
\left(\forall \varphi_{0} \in C_{0}:\left\|\varphi_{0}\right\|<\lambda\right): t_{0}+\sigma \in J^{+}\left(t_{0}, \varphi_{0}\right) \text { and } \\
\left(\forall t \geq t_{0}+\sigma, t \in J^{+}\left(t_{0}, \varphi_{0}\right)\right): \\
\left|x\left(t ; t_{0}, \varphi_{0}\right)\right|<\epsilon ;
\end{gathered}
$$

c) uniformly asymptotically stable if it is uniformly stable and uniformly attractive.

Definition 2: [3] We say that the function $V:\left[t_{0}, \infty\right) \times \mathbb{R}^{n} \rightarrow \mathbb{R}^{+}$belongs to the class $q_{0}$ if:

1. The function $V$ is continuous on $\bigcup_{k=1}^{\infty} G_{k}$ and $V(t, 0)=0$ for $t \in\left[t_{0}, \infty\right)$.

2. The function $V$ is Lipschitzian with respect to its second argument on each of the sets $G_{k}, k=1,2, \ldots$.

3. For each $k=1,2, \ldots$ and $\left(t_{0}^{*}, x_{0}^{*}\right) \in \sigma_{k}$, there exist the finite limits

$$
V\left(t_{0}^{*}-0, x_{0}^{*}\right)=\lim _{\substack{(t, x) \rightarrow\left(t_{0}^{*}, x_{0}^{*}\right) \\(t, x) \in G_{k}}} V(t, x), \quad V\left(t_{0}^{*}+0, x_{0}^{*}\right)=\lim _{\substack{(t, x) \rightarrow\left(t_{0}^{*}, x_{0}^{*}\right) \\(t, x) \in G_{k+1}}} V(t, x) .
$$


4. The following equality holds:

$$
V\left(t_{0}^{*}-0, x_{0}^{*}\right)=V\left(t_{0}^{*}, x_{0}^{*}\right)
$$

In the sequel, we shall use the following functional classes, assuming that conditions $\mathrm{H} 1, \mathrm{H} 2$ and $\mathrm{H} 3$ are met:

$P C\left[\left[t_{0}, \infty\right), \mathbb{R}^{n}\right]=\left\{x:\left[t_{0}, \infty\right) \rightarrow \mathbb{R}^{n}: x(t)\right.$ is piecewise continuous with points of discontinuity of the first kind (i.e., the left and right limits exist there, and they are bounded) on the interval $\left(t_{0}, \infty\right)$ at which it is left continuous $\}$;

$$
\Omega=\left\{x \in P C\left[\left[t_{0}, \infty\right), \mathbb{R}^{n}\right]: V(s, x(s)) \leq V(t, x(t)), t-h \leq s \leq t, t \geq t_{0}, V \in q_{0}\right\} .
$$

Let $V \in q_{0}, x \in P C\left[\left[t_{0}, \infty\right), \mathbb{R}^{n}\right]$, and $\left.t \neq \tau_{k}(x)\right), k=1,2, \ldots$

Introduce the function

$$
\dot{V}(t, x(t))=\frac{\partial V}{\partial t}+\frac{\partial V}{\partial x}[A(t) x(t)+B(t) x(t-h)] .
$$

Let $t_{1}, t_{2}, \ldots\left(t_{0}<t_{1}<t_{2}<\ldots\right)$ are the moments at which the integral curve $\left(t, x\left(t ; t_{0}, \varphi_{0}\right)\right)$ of (1-3) crosses the hypersurfaces $\sigma_{k}, k=1,2, \ldots$.

Remark 1: Let us note that conditions $\mathrm{H} 1-\mathrm{H} 4$ and $\mathrm{H} 7$ imply that $t_{k} \rightarrow \infty$ as $k \rightarrow \infty$ and $J^{+}\left(t_{0}, \varphi_{0}\right)=\left[t_{0}, \infty\right)$.

In proving the main results of the paper we shall use the following statements:

Theorem 1: Let the following assumptions hold:

1. Conditions $\mathrm{H} 1-\mathrm{H} 4$ and $\mathrm{H} 7$ are fulfilled.

2. $g \in P C\left[\left[t_{0}, \infty\right) \times \mathbb{R}_{+}, \mathbb{R}_{+}\right]$and $g(t, 0)=0$ for $t \in\left[t_{0}, \infty\right)$.

3. $B_{k} \in C\left[\mathbb{R}_{+}, \mathbb{R}_{+}\right], \stackrel{B}{k}_{k}(0)=0$ and the functions $\psi_{k}: \mathbb{R}_{+} \rightarrow \mathbb{R}_{+}, \quad \psi_{k}(u)=$ $u+B_{k}(u)$ are nondecreasing with respect to $u, k=1,2, \ldots$.

4. The maximal solution $r\left(t ; t_{0}, u_{0}\right)$ of the problem

$$
\begin{gathered}
\dot{u}=g(t, u), t \neq t_{k}, \quad k=1,2, \ldots, \\
u\left(t_{0}+0\right)=u_{0} \geq 0 \\
\Delta u\left(t_{k}\right)=B_{k}\left(u\left(t_{k}\right)\right), \quad k=1,2, \ldots
\end{gathered}
$$

is defined on the interval $\left[t_{0}, \infty\right)$.

5. The function $V \in \mathbb{V}_{0}$ is such that

$$
V\left(t_{0}, \varphi_{0}\left(t_{0}\right)\right) \leq u_{0}
$$

and the inequalities

$$
\begin{aligned}
& \dot{V}(t, x(t)) \leq g(t, V(t, x(t))), t \neq \tau_{k}(x(t)), k=1,2, \ldots \\
& V\left(t+0, x(t)+C_{k} x(t)\right) \leq \psi_{k}(V(t, x(t))), t=\tau_{k}(x(t)), k=1,2, \ldots \\
& \text { are satisfied for } t \geq t_{0} \text { and } x \in \Omega .
\end{aligned}
$$




$$
V\left(t, x\left(t ; t_{0}, \varphi\right)\right) \leq r\left(t ; t_{0}, u_{0}\right), \quad t \in\left[t_{0}, \infty\right)
$$
by

Proof: The maximal solution $r\left(t ; t_{0}, u_{0}\right)$ of problem (4) for $t \in\left(t_{0}, \infty\right)$ is defined

$$
r\left(t ; t_{0}, u_{0}\right)=\left\{\begin{array}{cc}
r_{0}\left(t ; t_{0}, u_{0}^{+}\right), & t_{0}<t \leq t_{1}, \\
r_{1}\left(t ; t_{1}, u_{1}^{+}\right), & t_{1}<t \leq t_{2}, \\
\ldots \ldots \ldots, & \\
r_{k}\left(t ; t_{k}, u_{k}^{+}\right), & t_{k}<t \leq t_{k+1}, \\
\ldots \ldots \ldots, &
\end{array}\right.
$$

where $r_{k}\left(t ; t_{k}, u_{k}^{+}\right)$is the maximal solution of the equation $\dot{u}=g(t, u)$ without impulses, that is defined on the interval $\left(t_{k}, t_{k+1}\right], k=0,1,2, \ldots$, for which $u_{k}^{+}=$ $\psi_{k}\left(r_{k-1}\left(t_{k} ; t_{k-1}, u_{k-1}^{+}\right)\right), k=1,2, \ldots$ and $u_{0}^{+}=u_{0}$.

Let $t \in\left(t_{0}, t_{1}\right]$. Then, it follows from the corresponding comparison theorem in the continuous case [1], that

$$
V\left(t, x\left(t ; t_{0}, \varphi_{0}\right)\right) \leq r\left(t ; t_{0}, u_{0}\right)
$$

i.e., inequality (7) is fulfilled for $t \in\left(t_{0}, t_{1}\right)$.

Let us suppose that (7) holds true for $t \in\left(t_{k-1}, t_{k}\right], k>1$. Then, using (6) and the fact that the function $\psi_{k}$ is nondecreasing, we obtain

$$
\begin{aligned}
& V\left(t_{k}+0, x\left(t_{k}+0 ; t_{0}, \varphi_{0}\right)\right) \leq \psi_{k}\left(V\left(t_{k}, x\left(t_{k} ; t_{0}, \varphi\right)\right)\right) \\
& \leq \psi_{k}\left(r\left(t_{k} ; t_{0}, u_{0}\right)\right)=\psi_{k}\left(r_{k}\left(t_{k} ; t_{k-1}, u_{k-1}^{+}\right)\right)=u_{k}^{+} .
\end{aligned}
$$

We apply the comparison theorem from [1] again for $t \in\left(t_{k}, t_{k+1}\right]$ and obtain

$$
V\left(t, x\left(t ; t_{0}, \varphi_{0}\right)\right) \leq r_{k}\left(t ; t_{k}, u_{k}^{+}\right)=r\left(t ; t_{0}, u_{0}\right),
$$

i.e., inequality (7) is satisfied for $t \in\left(t_{k}, t_{k+1}\right]$. The proof is completed by induction.

Corollary 1: Let the following assumptions hold:

1. Conditions $\mathrm{H} 1-\mathrm{H} 4$ and $\mathrm{H} 7$ are met.

2. The function $V \in \mathbb{q}_{0}$ is such that the inequalities

$$
\begin{gathered}
\dot{V}(t, x(t)) \leq 0, \quad t \neq \tau_{k}(x(t)), k=1,2, \ldots, \\
V\left(t+0, x(t)+C_{k} x(t)\right) \leq V(t, x(t)), \quad t=\tau_{k}(x(t)), k=1,2, \ldots
\end{gathered}
$$

Then

are valid for $t \geq t_{0}$ and $x \in \Omega$.

$$
V\left(t, x\left(t ; t_{0}, \varphi_{0}\right)\right) \leq V\left(t_{0}, \varphi_{0}\left(t_{0}\right)\right), \quad t \in\left[t_{0}, \infty\right)
$$




\section{Main Results}

Theorem 2: Let the following conditions hold:

1. Conditions H1-H7 are met.

2. The elements of the matrix-valued $n \times n$-function $B(t)$ are nonpositive for all $t \in\left(t_{0}, \infty\right)$.

Then the zero solution of system (1), (2) is uniformly stable.

Proof: Let $\epsilon>0$ be chosen arbitrarily. We choose $\delta=\delta(\epsilon)>0$ such that $\delta<\epsilon$.

Let $\varphi_{0} \in C_{0}:\left\|\varphi_{0}\right\|<\delta$ and let $x(t)=x\left(t ; t_{0}, \varphi_{0}\right)$ be a solution to problem (1-3).

We define the function $V(t, x)=\langle x, x\rangle=x^{T} x$. Then the set $\Omega$ is defined by the equality

$$
\Omega=\left\{x \in P C\left[\left[t_{0}, \infty\right), \mathbb{R}^{n}\right]:\langle x(s), x(s)\rangle \leq\langle x(t), x(t)\rangle, t-h \leq s \leq t, t \geq t_{0}\right\} .
$$

Now we shall estimate $\dot{V}(t, x(t))$ for $t \in\left(t_{0}, \infty\right), t \neq \tau_{k}(x(t))$, and $x \in \Omega$. It follows from condition $\mathrm{H} 5$ and condition 2 of Theorem 2 that

$$
\begin{gathered}
\dot{V}(t, x(t))=\dot{x}^{T}(t) x(t)+x^{T}(t) \dot{x}(t) \\
=[A(t) x(t)+B(t) x(t-h)]^{T} x(t)+x^{T}(t)[A(t) x(t)+B(t) x(t-h)] \\
=x^{T}(t)\left[A^{T}(t)+A(t)\right] x(t)+x^{T}(t-h) B^{T}(t) x(t) \\
+x^{T}(t) B(t) x(t-h)=2\langle x(t) B(t), x(t-h)\rangle \\
\leq 2\langle x(t) B(t), x(t)\rangle \leq 0 .
\end{gathered}
$$

Let $t=\tau_{k}(x(t))$. After using $\mathrm{H} 6$ we obtain

$$
V\left(t+0, x(t)+C_{k} x(t)\right)=\sum_{k=1}^{n}\left(1+c_{k i}\right)^{2} x^{2}\left(t_{k}\right) \leq V(t, x(t)), k=1,2, \ldots .
$$

Hence, the conditions of Corollary 1 are met, and therefore,

$$
V\left(t, x\left(t ; t_{0}, \varphi\right)\right) \leq V\left(t_{0}, \varphi_{0}\left(t_{0}\right)\right), \quad t \in\left(t_{0}, \infty\right)
$$

i.e.,

$$
\left|x\left(t ; t_{0}, \varphi_{0}\right)\right|^{2} \leq\left|\varphi_{0}\left(t_{0}\right)\right|^{2}, \quad t \in\left(t_{0}, \infty\right)
$$

The last inequality yields

$$
\left|x\left(t ; t_{0}, \varphi_{0}\right)\right|^{2} \leq\left|\varphi_{0}\left(t_{0}\right)\right|^{2} \leq\left\|\varphi_{0}\right\|^{2}<\delta^{2}<\epsilon^{2}
$$

whence $\mid x\left(t ; t_{0}, \varphi_{0}\right)<\epsilon$ for $t \in\left(t_{0}, \infty\right)$. This proves the uniform stability of the solution $x(t) \equiv 0$ of system (1), (2).

Theorem 3: Let the following conditions hold:

1. Conditions $\mathrm{H} 1-\mathrm{H} 7$ are met.

2. $\quad B(t)=\operatorname{diag}\left(b_{1}(t), \ldots, b_{n}(t)\right)$ and $b_{k}(t) \leq-\gamma_{k}<0, k=1,2, \ldots$ 
Then the zero solution of system (1), (2) is uniformly asymptotically stable.

Proof: We consider the function $V(t, x)=\langle x, x\rangle$. Analogously to (8), we obtain the estimate

$$
\dot{V}(t, x(t)) \leq 2\langle x(t) B(t), x(t)\rangle
$$

for $t \geq t_{0}, t \neq \tau_{k}(x(t))$ and $x \in \Omega$.

Let $\gamma=\min \gamma_{k}, k=1, \ldots, n$. Then, it follows from condition 2 of Theorem 3 and from (9) that

$$
\dot{V}(t, x(t)) \leq-2 \gamma|x(t)|^{2}, \quad t>t_{0}, \quad t \neq \tau_{k}(x(t)), \quad x \in \Omega .
$$

Since the conditions of Theorem 2 are satisfied, it follows that the zero solution of system (1), (2) is uniformly stable.

Now we shall prove that the zero solution is uniformly attractive.

Let $\epsilon>0$ be arbitrary chosen. We take $\eta=\eta(\epsilon)>0$ such that $\eta<\epsilon$.

Let $\lambda=$ const $>0$ and $\sigma=\sigma(\epsilon)>0$ be such that $\sigma>\frac{\lambda^{2}}{2 \gamma \eta^{2}}$. $3)$.

Let $\varphi_{0} \in C_{0},\left\|\varphi_{0}\right\|<\lambda$ and $x(t)=x\left(t ; t_{0}, \varphi_{0}\right)$ be the solution of the problem (1-

If we assume that $\left|x\left(t ; t_{0}, \varphi_{0}\right)\right| \geq \eta$ for $t \in\left[t_{0}, t_{0}+\sigma\right]$, then (10) implies the inequalities

$$
\begin{aligned}
& V\left(t, x\left(t ; t_{0}, \varphi_{0}\right)\right) \leq V\left(t_{0}, \varphi_{0}\left(t_{0}\right)\right)-\int_{t_{0}}^{t} 2 \gamma|x(s)|^{2} d s \\
& \leq\left\|\varphi_{0}\right\|^{2}-2 \gamma \int_{t_{0}}^{t}|x(s)|^{2} d s \leq \lambda^{2}-2 \gamma \eta^{2} \sigma<0
\end{aligned}
$$

which contradict to the choice of the function $V(t, x) \in \mathbb{q}_{0}$.

Hence, there exists a $t^{*} \in\left[t_{0}, t_{0}+\sigma\right]$ such that $x\left(t^{*} ; t_{0}, \varphi_{0}\right) \mid<\eta$. Thus, by virtue of Corollary 1 for $t \geq t^{*}$ (as well as for $t \geq t_{0}+\sigma$ ), the inequality

$$
V\left(t, x\left(t ; t_{0}, \varphi_{0}\right)\right) \leq V\left(t^{*}, x\left(t^{*} ; t_{0}, \varphi_{0}\right)\right)
$$

is valid, i.e., $\left|x\left(t ; t_{0}, \varphi_{0}\right)\right|^{2} \leq\left|x\left(t^{*} ; t_{0}, \varphi_{0}\right)\right|^{2}, t \geq t^{*}$.

The last inequality implies the inequalities

$$
\left|x\left(t ; t_{0}, \varphi_{0}\right)\right|^{2} \leq\left|x\left(t^{*} ; t_{0}, \varphi_{0}\right)\right|^{2}<\eta^{2}<\epsilon^{2}, t \geq t_{0}+\sigma
$$

The last inequalities show that the zero solution of system (1), (2) is uniformly attractive.

\section{Acknowledgement}

The present work was supported by the Bulgarian Ministry of Education, Science and Technologies under Grant MM-422. 
Drumi Bainov is grateful to the American National Research Council and Office for Central Europe and Eurasia, for their financial support during his stay at the University of Southwestern Louisiana, Lafayette, where this work was carried out.

\section{References}

[1] Lakshmikantham, V., Leela, S. and Martynyuk, A.A., Stability Analysis of Nonlinear Systems, Marcel Dekker, Inc., New York 1989.

[2] Lakshmikantham, V. and Rama Mohana Rao, M., Integro-differential equations and extension of Lyapunov's method, J. Math. Anal. Appl. 30 (1970), 435-447.

[3] Simeonov, P.S. and Bainov, D.D., Stability with respect to part of the variables in systems with impulse effect, J. Math. Anal. Appl. 117 (1986), 247-263. 


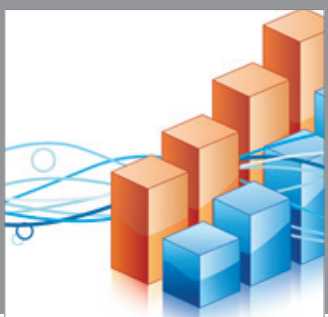

Advances in

Operations Research

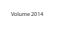

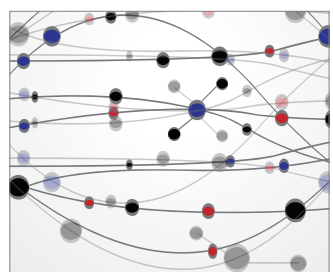

\section{The Scientific} World Journal
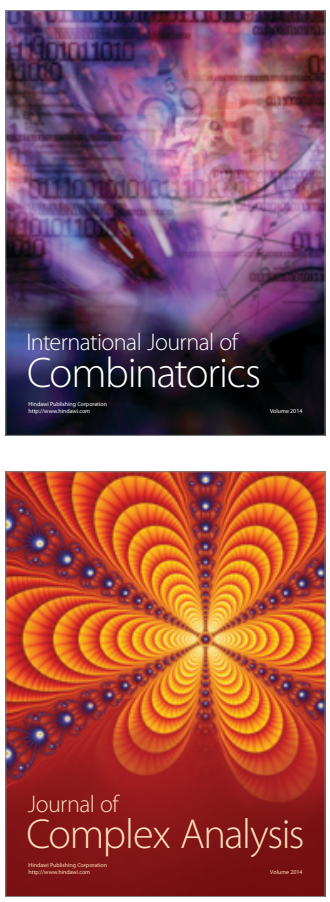

International Journal of

Mathematics and

Mathematical

Sciences
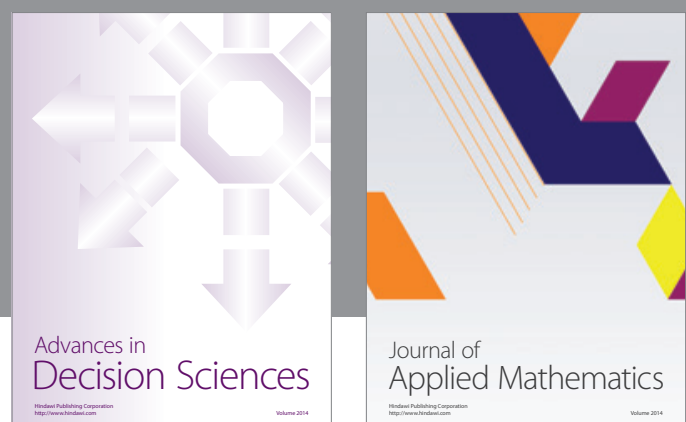

Journal of

Applied Mathematics
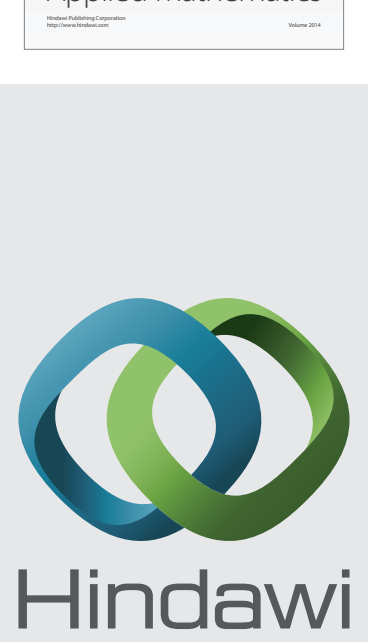

Submit your manuscripts at http://www.hindawi.com
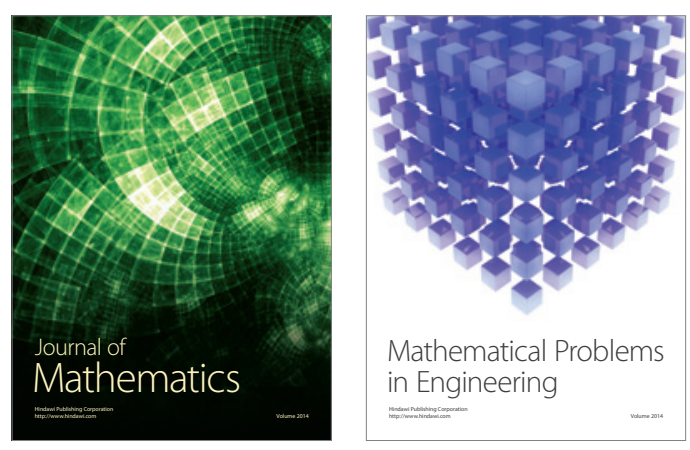

Mathematical Problems in Engineering
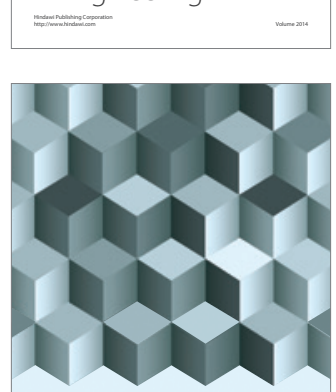

Journal of

Function Spaces
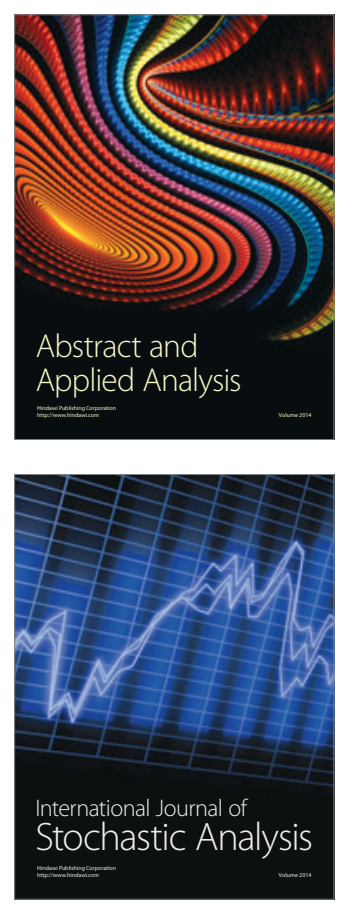

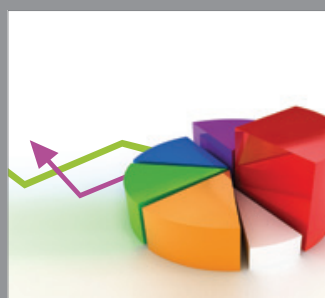

ournal of

Probability and Statistics

Promensencen
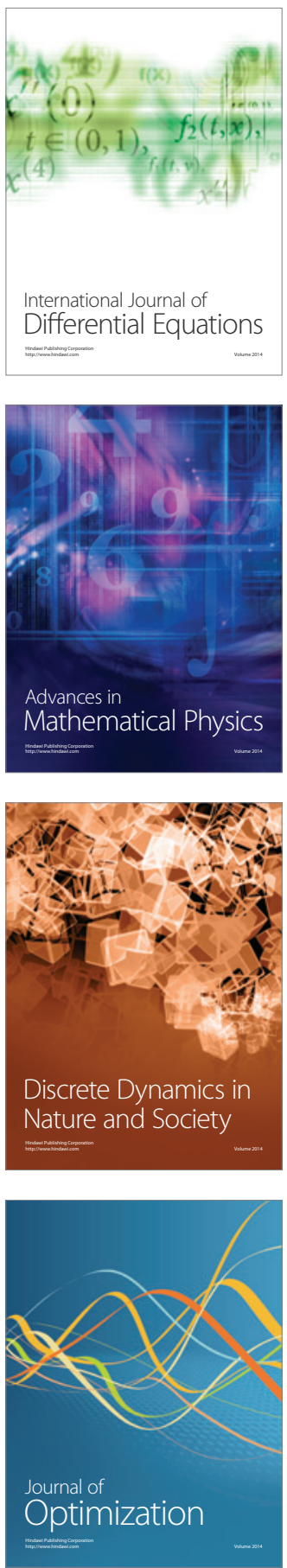